Article

\title{
Variable Wavefront Curvature Phase Retrieval Compared to Off-Axis Holography and Its Useful Application to Support Intraoperative Tissue Discrimination
}

\author{
Daniel Claus ${ }^{1,2, *} \mathbb{C}$, Jörg Hennenlotter ${ }^{3}$, Qi Liting ${ }^{1}$, Giancarlo Pedrini ${ }^{1}{ }^{\mathbb{C}}$, Arnulf Stenzl ${ }^{3}$ and \\ Wolfgang Osten ${ }^{1}$ \\ 1 Institut für Technische Optik, Universität Stuttgart, Pfaffenwaldring 9, 70569 Stuttgart, Germany; \\ st146385@stud.uni-stuttgart.de (Q.L.); pedrini@ito.uni-stuttgart.de (G.P.); osten@ito.uni-stuttgart.de (W.O.) \\ 2 Institut für Lasertechnologien in der Medizin und Messtechnik, Helmholtzstrasse 12, 89081 Ulm, Germany \\ 3 Universitätsklinik für Urologie, Eberhard Karls Universität Tübingen, Hoppe-Seyler Straße 3, \\ 72076 Tübingen, Germany; joerg.hennenlotter@med.uni-tuebingen.de (J.H.); \\ urologie@med.uni-tuebingen.de (A.S.) \\ * Correspondence: daniel.claus@ilm-ulm.de or claus@ito.uni-stuttgart.de; Tel.: +49-(0)73-1142-9225
}

Received: 11 September 2018; Accepted: 28 October 2018; Published: 3 November 2018

check for updates

Featured Application: Phase retrieval via variable wave front curvature for intraoperative medical imaging.

\begin{abstract}
Quantitative phase imaging can reveal morphological features without having to stain the biological sample. This property has important implications for intraoperative applications since the time spent during histopathology can be reduced from a few minutes to a few seconds. However, most common quantitative phase imaging techniques are based on the interferometric principle, which makes them more prone to disturbing environmental influences, such as temperature drift and air turbulence. In the last decade, with the advance of computing power, many different iterative quantitative phase imaging techniques, which only require the recording of the diffracted wavefield, and therefore offer increased robustness towards environmental disturbances, have been proposed. These are particularly well-suited for the application outside the well-controlled lab environment such as an operating theatre. The optical performance of our developed iterative phase retrieval method based on variable wavefront curvature will be evaluated by reference to off-axis digital holography and applied for intraoperative discrimination of tissue.
\end{abstract}

Keywords: phase retrieval; quantitative phase imaging; biomedical imaging

\section{Introduction}

Quantitative phase imaging is particularly useful for the investigation of biological thin tissue sections, which are generally transparent and hence do not show high amplitude contrast in a conventional optical microscope. It is therefore not easy to visualize such cells unless a staining is applied, which is time-consuming and may harm or even kill the cell and hence change their natural behavior. For translucent samples, the scattered light (which contains all the structural information about the object) is $90^{\circ}$ out of phase to the unscattered light. When the object is weak (first Born approximation), this means that the modulus of the image (hence its intensity) is negligibly affected by the scattered waves. Conventional Zernike phase contrast microscopy [1] partly resolves this problem by reducing the amplitude of the undiffracted light and by introducing an additional $90^{\circ}$ phase shift 
into the diffracted light. In the image, both terms (scattered and unscattered) now interfere strongly (they are in phase or anti-phase with one another), greatly increasing the visibility of the specimen. However, conventional Zernike phase contrast microscopy is not quantitative and suffers from artifacts, such as "halos," which surround the edges of phase structures in the specimen and result in a reduced resolution. Other phase contrast imaging techniques such as differential interference contrast (DIC) do not suffer under loss of resolution, but DIC still does not yet result in quantitative phase imaging. In fact, DIC is a two-beam interference method with a lateral shear that approximately matches the microscope's resolution. It is hence sensitive to the phase gradient. Attempts have been made to recover the quantitative phase using spatial light modulators as an adjustable phase delay unit in Zernike phase contrast microscopy [2], called Spatial Light Interference Microscopy (SLIM) with the delay applied to phase ring, or in DIC, called Gradient Light Interference Microscopy (GLIM) [3] with the delay applied in the Fourier domain followed by an integration of the recovered phase gradient. However, SLIM still suffers from halo artifacts and loss of resolution, and GLIM suffers from the lack of directional sensitivity, which in detail means that it is insensitive to structural details oriented in a direction perpendicular to the shear direction. Instead of constant phases steps, various different shears have been applied in Reference [4] in order to recover the phase quantitatively.

On the other hand, the well-established digital holography only works well outside the laboratory environment if measures, such as pulsed laser light (freeze the motion in time) or common path geometries (both arms experience same environment), are applied in order to reduce the impact of disturbing environmental conditions on the recorded hologram. In rare cases of single-cell imaging can Gabor holography or lateral shearing interferometry be applied to recover the phase quantitatively. In these particular cases, the specimen only covers a small fraction of the field of view. Hence, the specimen region can by means of lateral shearing be brought into interference with a neighboring region, where light has passed through in an undiffracted manner. This approach offers a robust and compact solution to recover the phase. However, it is limited to the imaging of a few cells only. Instead, our endeavor is the imaging of thin tissue in order to discard the labeling in histopathology.

Iterative quantitative phase imaging can cover the aforementioned shortcomings via the recording of multiple diffraction patterns, which are then computer processed in an iterative manner to recover the quantitative phase. A key to phase retrieval imaging is the recording of the same information on multiple speckle decorrelated patterns. Many techniques have been developed based on this principle, such as the recording of two or more diffraction patterns at different planes [5]. In Reference [6], an aperture is moved across the object and the corresponding diffraction patterns are sequentially recorded. A phase mask has been applied to modulate the wavefront in References $[7,8]$ and the phase information could be recovered from diffraction patterns recorded at different wavelengths. The latter suffers from the problem of dispersion effects that result in different responses of the object under investigation with respect to the wavelength employed. Therefore, each of the recorded wavelength's corresponding speckle patterns may hold different information, which hinders or makes the application of the iterative phase retrieval approach impossible. In Reference [9], not the distance between sensor and object but the distance between point source and object was changed. Hence, compared to Reference [5], the lateral resolution did not suffer since it was the same for all diffraction patterns recorded. Moreover, the retrieval algorithms could be simplified via the application of the direct Fresnel transform.

In this paper, the optical performance of the curved illumination phase retrieval algorithm with regard to the optical resolution, phase sensitivity, and signal to noise ratio (SNR) was investigated and compared to digital off-axis holography using the same sensor and same distance to the object. Furthermore, the curved illumination phase retrieval method was applied for the label-free discrimination of cancerous and healthy human renal tissue. 


\section{Experimental Setup}

The experimental setup for both systems, iterative phase retrieval and digital holography, was configured on a breadboard in order to enable transportation to our medical partners in Tübingen to investigate human samples on site. The setup is shown in Figure 1. Light is launched from a HeNe Laser (Spectra-Physics, Irvine, CA, USA) $(632.83 \mathrm{~nm})$ into a single mode fibre coupler. One end of the fibre coupler is used as a point source. The wavelength and the distance to the object define the illumination function impinging upon the object. An aperture is positioned in close proximity to the object in order to restrict the field of view to ensure that the frequencies in the detector plane are below the Nyquist limit. The object-diffracted light and the reference wave are combined via a beam splitter cube of $20 \mathrm{~mm}$ length (Thorlabs BS016, 50:50 Non-Polarizing Beamsplitter Cube, 400-700 nm, Thorlabs, Newton, NJ, USA) and directed at slightly different angles to the sensor, at which an off-axis digital hologram is recorded. The distance between the illumination point source and the object is $85 \mathrm{~mm}$. The sensor employed is Ximea Modell MQ013RG-E2 $(1280 \times 1024,5.3 \mu \mathrm{m}$ pixel size). For the iterative phase retrieval system, the reference fibre is removed from the setup and the motorized actuator (Physik Instrumente M125.11, Physik Instrumente, Karlsruhe, Germany) holding the point source is axially displaced to different predefined positions to ensure the recording of speckle decorrelated diffraction patterns, as pointed out in Reference [9]. For the sake of a fair comparison, the beam splitter cube was not removed between the two different experiments, which resulted in the same effect on the object beam and its wavefront. Due to the smaller refraction angle in the glass (NBK7), the divergent wavefronts leaving the beam splitter have a stronger curvature compared to an equivalent passage of light through air. Therefore, the interference pattern appears to have been recorded from a shorter recording distance than the geometrical path length. Considering the refractive index of glass, the interferometrically recorded path length can be calculated, which we from now on refer to as virtual path length:

$$
l_{\text {vir }}=l_{\text {geo }} / n
$$

The actuator and the sensor are controlled using the in-house developed software ITOM [10], which enables an automated procedure.

At first, the resolution was investigated employing a United States Air Force resolution test target. The sensor was positioned at a virtual path length distance of $93 \mathrm{~mm}$ with respect to the object, and the point source at distances ranging from $73 \mathrm{~mm}$ to $85 \mathrm{~mm}$ with respect to the object. In accordance with the Nyquist criterion:

$$
X=\frac{\lambda d_{r e c}}{2 \Delta x \prime}
$$

The object size $X$ was restricted to $5.5 \mathrm{~mm}$, with $d_{\text {rec }}$ being the object to camera distance, and $\Delta x^{\prime}$ the sensor's pixel size. The minimum displacement $d_{\text {sp_axial }}$ applied to the actuator in order to obtain speckle decorrelation was:

$$
d_{s p \_a x i a l}=\frac{4 \lambda d_{i l l}^{2}}{X^{2}}
$$

which was equal to $0.6 \mathrm{~mm}$, with $\lambda$ being the wavelength $(632.83 \mathrm{~nm}), d_{\text {ill }}$ the longest illumination distance $(85 \mathrm{~mm})$ and $X$ being the object size. Speckle represented high-frequency information, which hence decorrelated quicker than low-frequency components.

In order to likewise obtain sufficient decorrelation of low-frequency components, the available displacement of the actuator was employed, with the recording of 10 diffraction patterns separated by $1.33 \mathrm{~mm}$. For the reconstruction, the combined distance $d_{\text {comb }}$ of the illumination and recording scheme was used in the implementation of the direct Fresnel propagation:

$$
d_{c o m b}=\frac{d_{i l l} d_{r e c}}{d_{i l l}+d_{r e c}}
$$




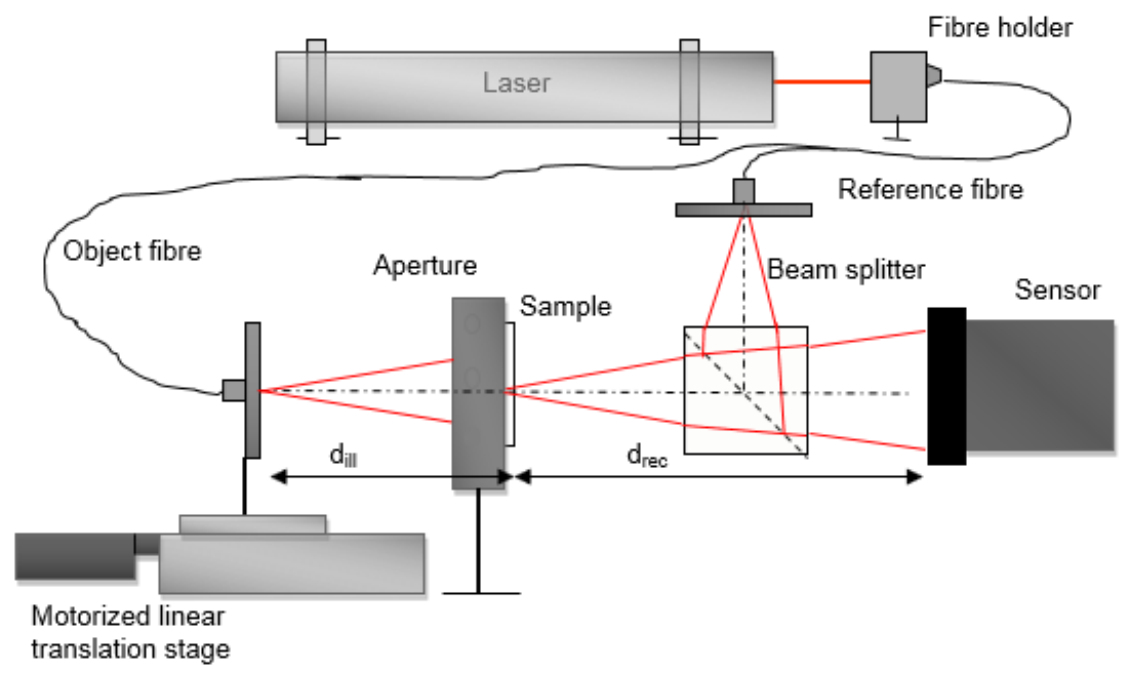

(a)

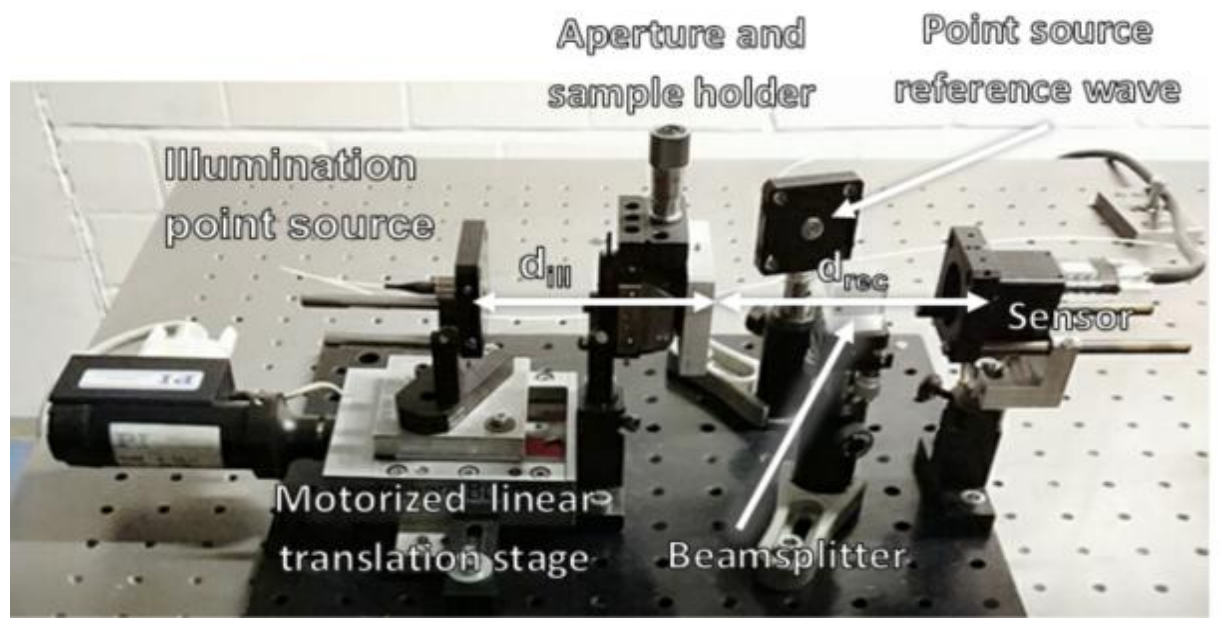

(b)

Figure 1. Opto-mechanical setup used for the comparison of the digital holography and iterative phase retrieval via curved illumination: (a) schematic setup, and (b) experimentally implemented setup.

The application of illumination sources with different curvature acts like a magnification to the recorded diffraction pattern, and in that manner, resulting in a changed pixel size $\overline{\Delta x^{\prime}}$ that will have to be applied in the reconstruction instead of the sensor's pixel size $\Delta x^{\prime}$ :

$$
\overline{\Delta x \prime}=\frac{d_{i l l} \Delta x \prime}{d_{i l l}+d_{r e c}}
$$

The relationship between the illumination distance and the resulting projected pixel size $\overline{\Delta x}$ has graphically been represented in Figure 2. However, the pixel size in the reconstruction plane when employing the direct Fresnel method is the same for all illumination distances as pointed out in Reference [9].

$$
\Delta x^{\prime \prime}=\frac{\lambda d_{r e c}}{N \Delta x \prime}
$$




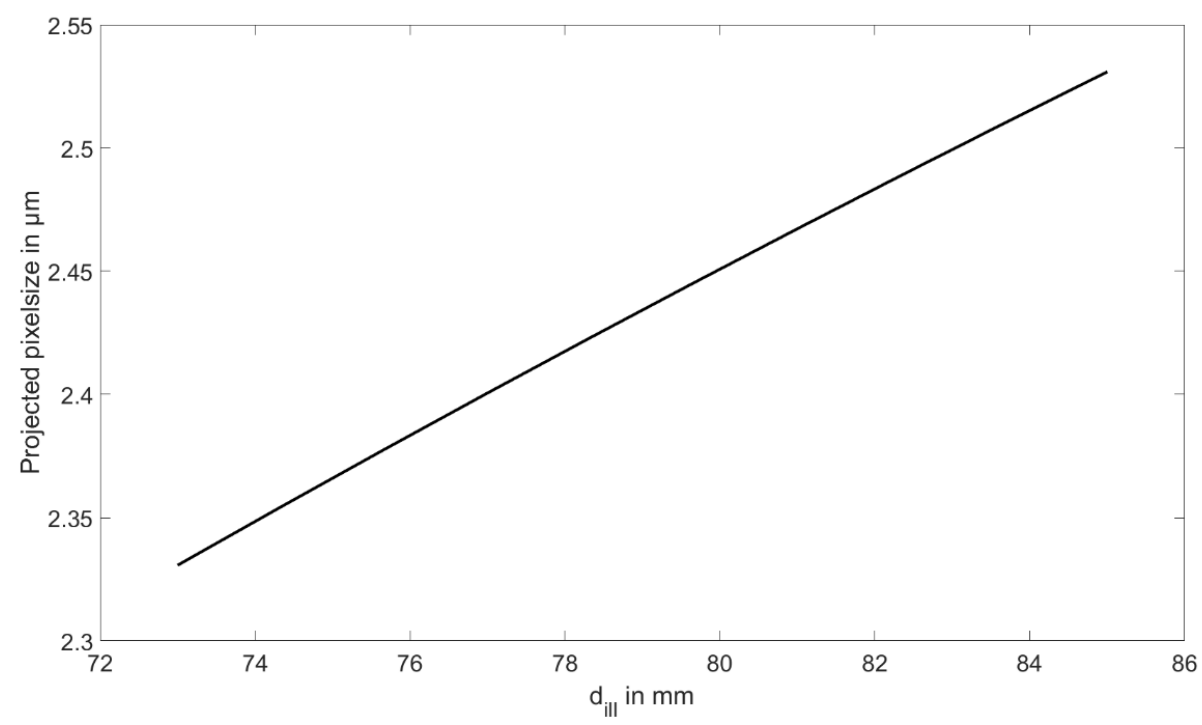

Figure 2. Change of projected pixel size $\overline{\Delta x}$ with respect to the object-illumination source distance.

The iterative approach was implemented in the conventional manner, replacing the recovered amplitude with the square root of the recorded intensity pattern at each plane while preserving the phase. In addition, Parseval's theorem was implemented to ensure the correct contribution of each diffraction pattern to the recovered complex wave.

The off-axis holograms were first Fourier filtered for the extraction of the image term only [11]. Afterwards, the numerical reconstruction was performed using the direct Fresnel method [11]. The reconstruction distance $d_{\text {recon }}$ that had to be employed, represents a function of the combined distance $d_{\text {comb }}$ and the reference source point distance $d_{\text {ref }}$ (with the virtual path length $d_{\text {ref }}=103 \mathrm{~mm}$ to the source of the reference wave):

$$
d_{\text {recon }}=\frac{d_{c o m b} \cdot d_{r e f}}{\left|d_{c o m b}-d_{r e f}\right|}
$$

Furthermore, the magnification adjusted pixel-size $\overline{\Delta x}$, see Equation (5), was used in the reconstruction process.

A recorded diffraction pattern and a recorded digital hologram are shown in Figure 3a,b, including a zoomed-in region at the bottom-right corner. The zoomed-in region highlights the effect of self-interference as encountered in the diffraction pattern and the interference with a separate reference wave, which arise at the off-axis hologram leading to the characteristic carrier frequency. The corresponding amplitude reconstructions are shown in Figure $3 c, d$. The averaged cross-section plots of the test target element that can still be resolved are shown at the bottom corner. The abscissa represents the pixel position and the ordinate represents the normalized averaged amplitude value. The resolution obtained has been evaluated with respect to Rayleigh's resolution criterion. Care was taken to ensure that the ratio between the smallest maximum and largest minimum of the investigated averaged test target element was always smaller than 0.81 . For the phase retrieval approach group 5 's 5 th and 6th element could be resolved for vertical and horizontal orientation, respectively, which corresponded to the recovery of structural details of $9.84 \mu \mathrm{m}$ and $8.77 \mu \mathrm{m}$ in width. Test target element 6 in group 5 could be resolved for both horizontal and vertical orientation when employing digital holography, which corresponded to resolving structural details of $8.77 \mu \mathrm{m}$ in width. In conclusion, the iterative approach and the phase retrieval method performed similarly well. 


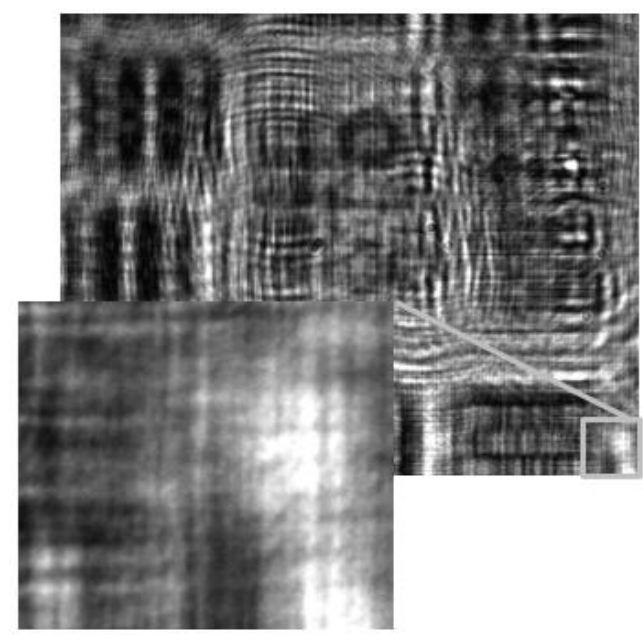

(a)

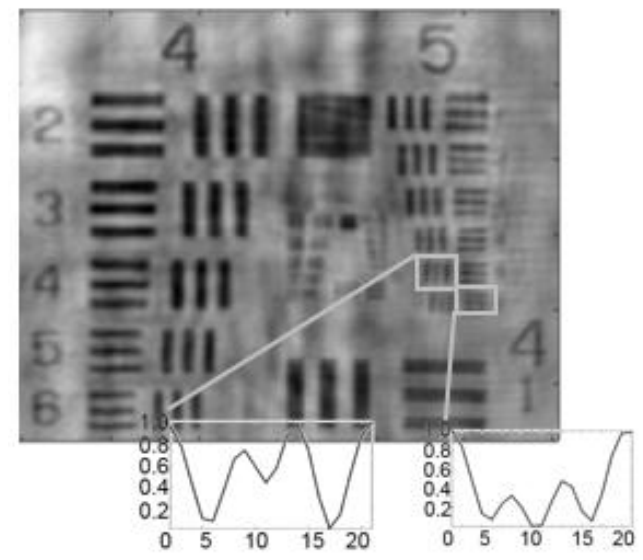

(c)

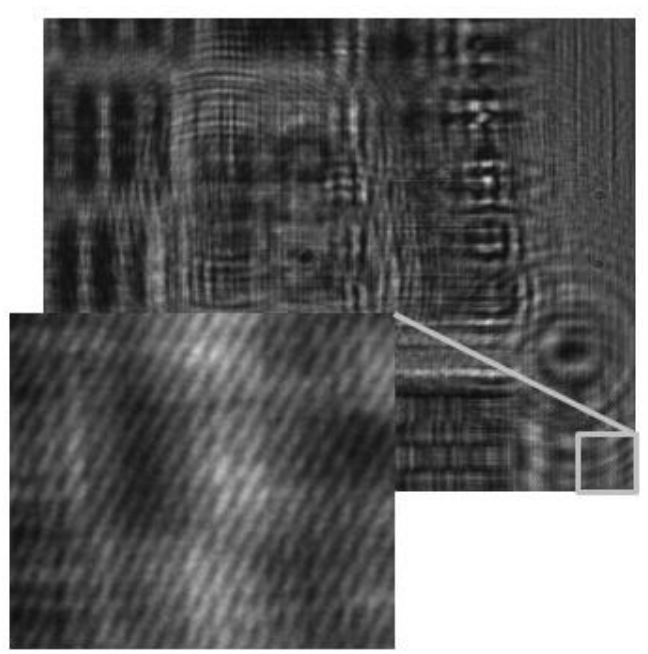

(b)

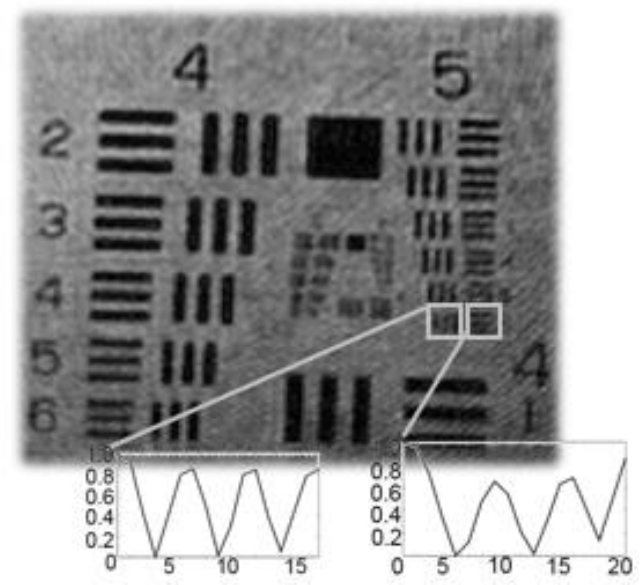

(d)

Figure 3. Recorded (a) diffraction pattern and (b) digital hologram with a conventional positive USAF test target serving as the object under investigation. The enlarged area in the bottom-right corner highlights the difference in the recording pattern caused by the carrier frequency introduced by the separate reference wave in off-axis digital holography. The corresponding amplitude reconstructions are shown in $(\mathbf{c}, \mathbf{d})$.

Besides the possibility to enable lensless imaging, the most prominent advantage of quantitative phase imaging is its high sensitivity to changes in the phase. At a next step, the phase sensitivity of the iterative phase retrieval method and off-axis digital holography have been compared (see Figure 4). For this purpose, a particularly designed and in-house manufactured fully transparent test target, with the elements etched into to the glass to a depth of $79.1 \mathrm{~nm}(L=\lambda / 8$ for $633 \mathrm{~nm}$ wavelength), was created. With an approximate refractive index $\mathrm{n}$ of the glass of 1.5 , the resulting phase step $\Delta \phi$ was:

$$
\Delta \phi=\frac{2 \pi L(n-1)}{\lambda}=\frac{\pi}{8}
$$




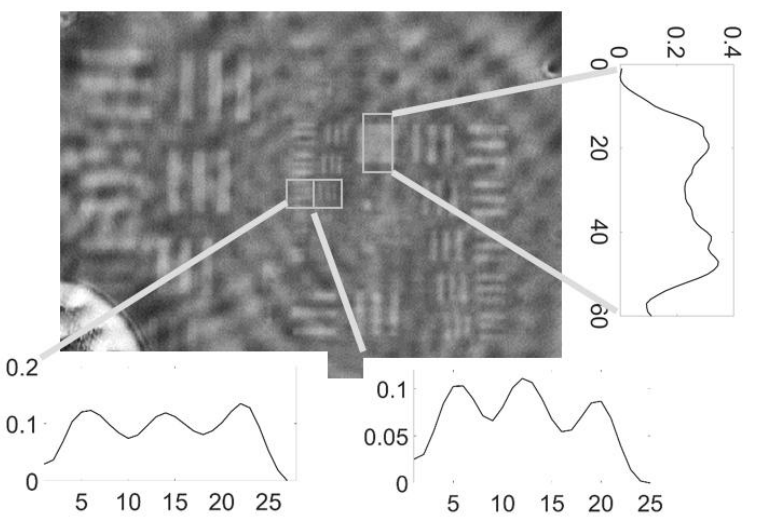

(a)

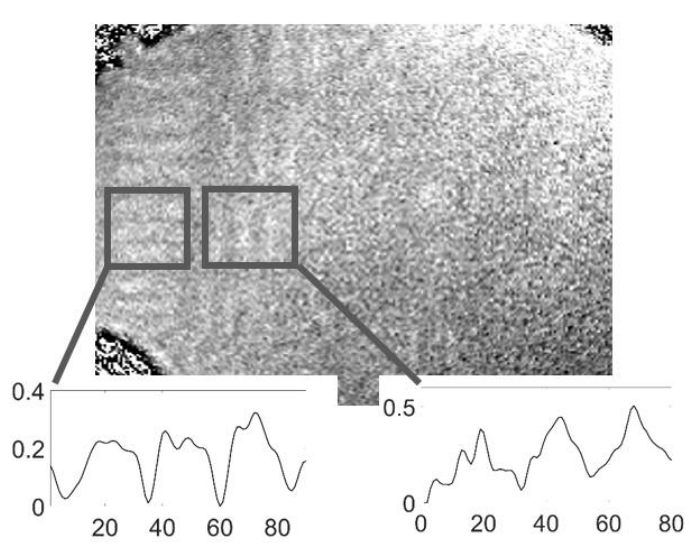

(b)

Figure 4. Phase reconstructions for a specifically engineered phase USAF test target with a phase step of $\pi / 4$ at $633 \mathrm{~nm}$ for the target elements: (a) iterative phase retrieval and (b) digital holography.

The resolution and the signal to noise ratio were used as objective quantitative measures in support of this comparison. It can be appreciated that the iterative phase retrieval approach performed significantly better in terms of resolution and signal to noise ratio. The abscissa in the graphs at the bottom of Figure $4 \mathrm{a}, \mathrm{b}$ correspond to the pixel position and the ordinate to phase in radians. Moreover, an averaged cross-section across the square can be seen at the left side of Figure $4 \mathrm{a}$, with the same parameter representation of the axis. The phase difference obtained for the square in comparison to its surrounding was $0.346 \mathrm{rad}$, which matched well with the expected phase step of $0.393 \mathrm{rad}$. For the iterative phase retrieval approach, the third element in group five could still be resolved $(40.3 \mathrm{lp} / \mathrm{mm}$, resulting in a smallest resolvable structure of $12.04 \mu \mathrm{m}$ in width), whereas for the digital off-axis hologram group 3 element 4 could only be resolved $(11.3 \mathrm{lp} / \mathrm{mm}$, resulting in a smallest resolvable structure of $44.19 \mu \mathrm{m}$ in width). The significantly improved resolution and signal to noise ratio for the phase retrieval approach in comparison to off-axis digital holography were due to the application of various speckle decorrelated diffraction patterns resulting in reducing speckle noise and different incident angles on the specimen, as in incoherent imaging [12]. According to Reference [13], the signal to noise ratio was calculated as the ratio between:

$$
\mathrm{SNR}=\frac{\mu}{\sigma}
$$

with $\mu$ representing the mean value and $\sigma$ the standard deviation. The SNR obtained for the digital holographic phase reconstruction was 6.08 and 22.78 for the quantitative phase image obtained via the iterative approach. Moreover, any environmental changes in the two interfering arms, caused for instance by heat, were likewise recorded and may hence negatively affect the result.

\section{Application to Medical Imaging}

After demonstrating the functionality of the iterative phase retrieval technique on a USAF test target, it was then applied for biomedical applications with the motivation to assist the discrimination between cancerous and normal tissue for histopathology, which currently necessitates the time-consuming staining process (roughly $15 \mathrm{~min}$ ) in order to visualize cell structures, by which the discrimination is made possible.

An intraoperative measurement technique based on quantitative phase imaging, which is sensitive to the slightest change in the propagation of the light due to refractive index changes, will reduce the time to a few seconds, which consequently results in decreasing costs and less stress/harm on the patient and the surgeon.

Three thin paraffine tissue sections of 5-7 $\mu \mathrm{m}$ thickness from cancerous and healthy renal tissue of oncologic surgeries have been investigated. Two different wavelengths have been employed to 
investigate those samples: $633 \mathrm{~nm}$ and $1064 \mathrm{~nm}$ (Nd:YAG) (see Figure 5). To track the convergence of the iterative algorithm, an error measure, represented by the root mean square difference between the measured diffraction pattern intensity and the iteratively retrieved intensity, was introduced. A quickly declining error measure over the number of iterations is shown in Figure $5 c$, which indicates a good convergence of the algorithms. The capital letters A, B, and C represent different diffraction planes.

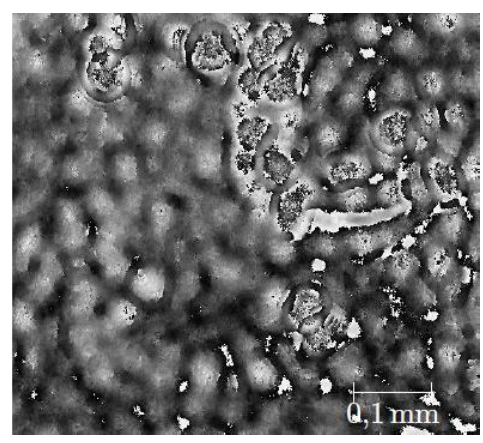

(a)

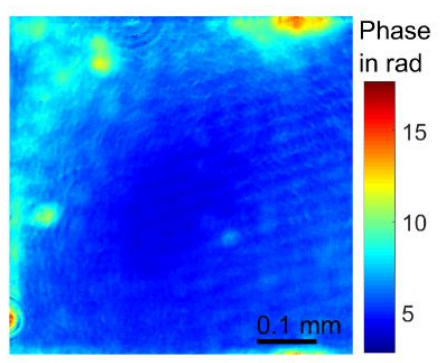

(d)

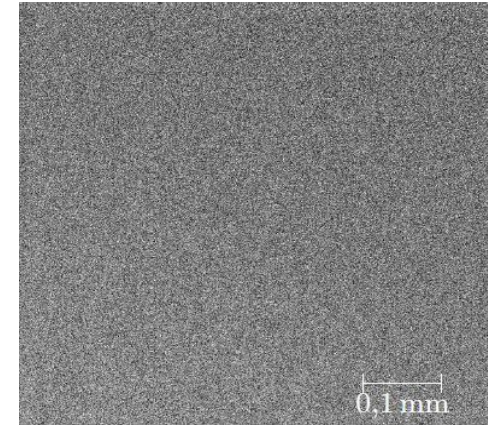

(b)

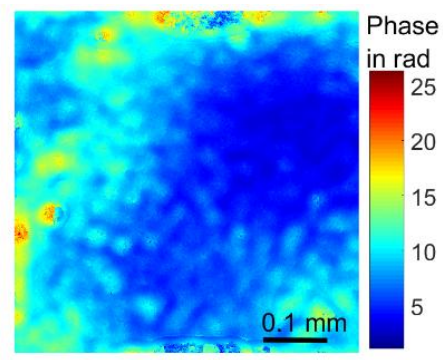

(e)

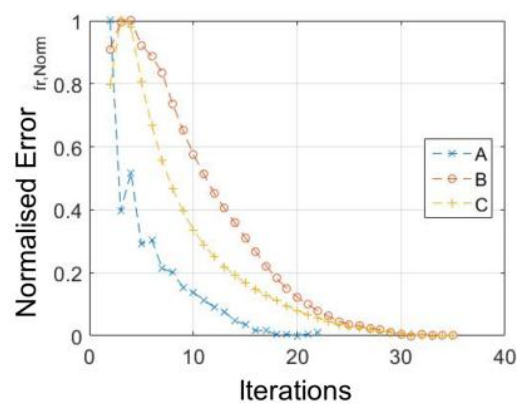

(c)

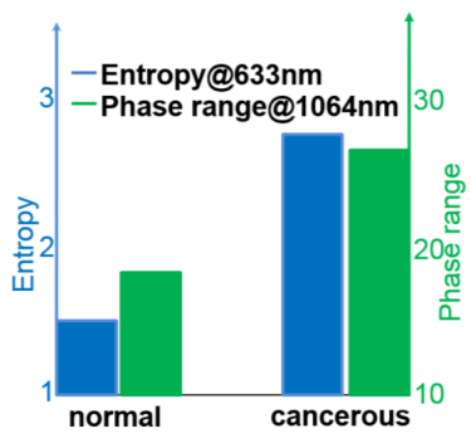

(f)

Figure 5. Phase reconstructions at $633 \mathrm{~nm}$ : (a) healthy, (b) cancer, (c) error measure curve for different diffraction planes $(A=4, B=6, C=8)$, and unwrapped phase reconstructions for: (d) healthy, (e) cancer, (f) entropy at $633 \mathrm{~nm}$ and phase range at $1064 \mathrm{~nm}$.

In Figure 5a,b, the quantitative phase reconstructions for normal and tumorous tissue are shown. For the healthy tissue, a smooth and almost regular phase could be reconstructed, which confirmed the findings in Reference [14], where a more regular structure was likewise reported for normal tissue. For the cancerous tissue, a noisy phase reconstruction was obtained for $633 \mathrm{~nm}$, which indicated either that cancerous tissue at such thickness consisted of more scattering layers and/or that cancerous tissue consisted of finer scattering features resulting in large scattering angles, which therefore cannot be recorded by the optical arrangement. The latter has been confirmed by various publications, where a more finely meshed extra cellular matrix has been reported for cancerous tissue in comparison to normal tissue $[15,16]$. For comparison, in the case of tumorous tissue, scans resulted in a noisy phase image due to multiple scattering events within the tissue (see Figure 5 b). A possible reason for the increased scattering is the more densely structured extracellular matrix and the hyper-vascularization in tumorous tissue. The changes in scattering behavior on a cellular level have already been reported in Reference [17]. Therefore, it is not too surprising that the changed scattering behavior can likewise be observed on tissue level. In this case, due to multiple scattering events on the cancerous tissue, a very strong difference can be appreciated between the two phase reconstructions.

In order to enable visualization of a smooth phase distribution for all samples, a Nd:YAG with $1064 \mathrm{~nm}$ was employed, by which the scattering coefficient according to Mie scattering could be reduced by a factor of 8 . This was clearly visible in the phase reconstructions. After unwrapping the obtained phase maps, an increase in phase delay was recognized, which indicated that the refractive 
index of the cancerous sample was increased in comparison to the healthy sample (all sections of the same thickness), see Figure 5d,e, which corresponds to the findings in Reference [14]. The entropy was used to evaluate the scattering at $633 \mathrm{~nm}$ and the phase range (difference between maximum and minimum phase value) at $1064 \mathrm{~nm}$. The corresponding values for both parameters have been plotted and are displayed in Figure $5 f$.

\section{Discussion}

The iterative phase retrieval method based on a changing curved illumination function was compared to the well-established digital off-axis holography. It was found that the resolution obtained could compete with digital off-axis holography and the phase sensitivity even outperformed digital off-axis holography. Due to this property, the iterative phase retrieval method was then applied to renal tissue discrimination. Preliminary results have been shown, which demonstrate its potential for intraoperative tumor diagnosis. Discrimination parameters could be derived from the increased scattering and phase delay of tumorous tissue in comparison to healthy tissue. At the moment, paraffin sections have been investigated, but future research will focus on the application of cryosections, which are quicker to prepare and enable the preservation of the tissue as close as possible to its natural state. Further investigations will involve tissue samples from the transition region between cancer and normal tissue, which medically is likewise very important for removing the entire malign tumor. Moreover, other tissue malignancies will be investigated in order to generalize the findings obtained. The results obtained will be compared with the gold standard in histopathology using stained samples and other imaging techniques.

This work was funded by the industry on campus project IoC105 "Intraoperative model-based identification of tissue properties using a multimodal and multiscale approach" and the H2020 European Project HOLO (TWINN 687328). The work conducted on human tissue has been performed in the scope of the ethics application number 154/2018BO2 listed at the Eberhard Karls Universität Tübingen.

Author Contributions: D.C. developed the idea and wrote the major part of the paper, Q.L. arranged and conducted the experiment with the help of G.P. and D.C., J.H. supplied the medical samples, provided medical expertise and helped writing the paper, A.S. and W.O. applied for the funding and managed the project.

Funding: This research was funded by the Industry on Campus project IoC105 under the title "Intraoperative model based identification of tissue properties using a multimodal and multiscale approach"

Conflicts of Interest: The authors declare no conflicts of interest.

\section{References}

1. Zernike, F. Phase contrast, a new method for the microscopic observation of transparent objects part I. Physica 1942, 9, 686-698. [CrossRef]

2. Wang, Z.; Millet, L.; Mir, M.; Ding, H.; Unarunotai, S.; Rogers, J.; Gillette, M.U.; Popescu, G. Spatial light interference microscopy (SLIM). Opt. Express 2011, 19, 1016-1026. [CrossRef] [PubMed]

3. Nguyen, T.H.; Kandel, M.E.; Rubessa, M.; Wheeler, M.B.; Popescu, G. Gradient light interference microscopy for 3D imaging of unlabeled specimens. Nat. Commun. 2017, 8, 1-9. [CrossRef] [PubMed]

4. Falldorf, C.; Agour, M.; Bergmann, R.B. Digital holography and quantitative phase contrast imaging using computational shear interferometry. Opt. Eng. 2015, 54, 024110. [CrossRef]

5. Almoro, P.; Pedrini, G.; Osten, W. Complete wavefront reconstruction using sequential intensity measurements of a volume speckle field. Appl. Opt. 2006, 45, 8596-8605. [CrossRef] [PubMed]

6. Faulkner, H.M.L.; Rodenburg, J.M. Movable Aperture Lensless Transmission Microscopy: A Novel Phase Retrieval Algorithm. Phys. Rev. Lett. 2004, 93, 023903. [CrossRef] [PubMed]

7. Zhang, F.; Pedrini, G.; Osten, W. Phase retrieval of arbitrary complex-valued fields through aperture-plane modulation. Phys. Rev. A 2007, 75, 043805. [CrossRef]

8. Bao, P.; Zhang, F; Pedrini, G.; Osten, W. Phase retrieval using multiple illumination wavelengths. Opt. Lett. 2008, 33, 309-311. [CrossRef] [PubMed] 
9. Claus, D.; Pedrini, G.; Osten, W. Iterative phase retrieval based on variable wave-front curvature. Appl. Opt. 2017, 56, F134-F137. [CrossRef] [PubMed]

10. Gronle, M.; Lyda, W.; Wilke, M.; Kohler, C.; Osten, W. Itom: An open source metrology, automation, and data evaluation software. Appl. Opt. 2014, 53, 2974-2982. [CrossRef] [PubMed]

11. Schnars, U.; Jueptner, W. Digital Holography: Digital Hologram Recording, Numerical Reconstruction, and Related Techniques; Springer: Berlin/Heidelberg, Germany, 2005.

12. Claus, D.; Pedrini, G. Ptychography: Quantitative Phase Imaging with Incoherent Imaging Properties; SPIE Photonics Europe: Strasbourg, France, 2018.

13. Smith, S.W. Statistics, Probability and Noise. In The Scientist and Engineer's Guide to Digital Signal Processing; California Technical Publishing: Poway, CA, USA, 1999; pp. 13-17.

14. Claus, D.; Maiden, A.M.; Zhang, F.; Sweeney, F.G.R.; Humphry, M.J.; Schluesener, H.; Rodenburg, J.M. Quantitative phase contrast optimised cancerous cell differentiation via ptychography. Opt. Express 2012, 20, 9911-9918. [CrossRef] [PubMed]

15. Chaudhuri, O.; Koshy, S.; da Cunha, C.B.; Shin, J.W.; Verbeke, C.S.; Allison, K.H.; Mooney, D. Extracellular matrix stiffness and composition jointly regulate the induction of malignant phenotypes in mammary epithelium. Nat. Mater. 2014, 13, 970-978. [CrossRef] [PubMed]

16. Lange, J.R.; Fabry, B. Cell and tissue mechanics in cell migration. Exp. Cell Res. 2013, 319, $2418-2423$. [CrossRef] [PubMed]

17. Richter, V.; Voit, F.; Kienle, A.; Schneckenburger, H. Light scattering microscopy with angular resolution and its possible application to apoptosis. J. Microsc. 2014, 257, 1-7. [CrossRef] [PubMed]

(C) 2018 by the authors. Licensee MDPI, Basel, Switzerland. This article is an open access article distributed under the terms and conditions of the Creative Commons Attribution (CC BY) license (http:// creativecommons.org/licenses/by/4.0/). 